\title{
A fluid-particle interaction method for blood flow with special emphasis on red blood cell aggregation
}

\author{
Tong Wang ${ }^{\mathrm{a}, *}$ and Zhongwen Xing ${ }^{\mathrm{b}}$ \\ ${ }^{a}$ Department of Mathematics, Nanjing University of Aeronautics and Astronautics \\ Nanjing, Jiangsu, 210016, China \\ ${ }^{\mathrm{b}}$ Department of Materials Science and Engineering, National Laboratory of Solid State \\ Microstructures, Nanjing University, Nanjing, Jiangsu, 210093, China
}

\begin{abstract}
This paper presents a fluid-particle interaction algorithm using the distributed Lagrange multiplier based fictitious domain method. The application of this method to the numerical investigation of motion and aggregation of red blood cells in two-dimensional microvessels is discussed. The cells are modelled as rigid biconcave-shaped neutrally buoyant particles. The aggregating force between two cells is derived from a Morse type potential function. The cell-cell interaction is coupled with the fluid-cell interaction through a time splitting scheme. Simulation results of multiple red blood cells in Poiseuille flow are presented. Because of its modular nature, this algorithm is applicable to a large class of problems involving the processes of particle aggregation and fluid-particle interaction.
\end{abstract}

Keywords: Fluid-particle interaction, red blood cells, aggregation, mathematical modelling

\section{Introduction}

Fluid-particle interaction is an example of time-dependent problems which have application in biomechanics and biological systems, e.g., blood flow in the cardiovascular system. The aim of this study is to simulate blood flow with blood cell aggregation in microvessels since aggregation of red blood cells (RBCs) plays a crucial role in many physiological phenomena. It has been observed that healthy RBCs disperse in the blood plasma. In some unhealthy blood or under some physiological conditions, rouleaux are formed which decreases the surface area of RBCs and increases the apparent viscosity of the blood, which severely reduces the amount of oxygen and nutrients that can be transported by RBCs. Because of their large volume fraction in blood and their aggregability, RBCs are the most important determinant of blood characteristics in microcirculation.

\footnotetext{
*Corresponding author: Tong Wang, Department of Mathematics, Nanjing University of Aeronautics and Astronautics, 29 Yudao Street, Nanjing, Jiangsu, 210016, China. Tel.: +86 25 84892440; Fax: +86 25 84498069; E-mail: twang@ nuaa.edu.cn.
}

0959-2989/14/\$27.50 @ 2014 - IOS Press and the authors. 
Numerical simulations can be a very powerful tool in providing insight into systems that are difficult to examine by in vivo or in vitro experiments, e.g., the aggregation of RBCs in microvessels [1-4]. In [2], the RBC aggregation kinetics is simulated using a particle method and the RBCs are modelled as rigid spheres. In [3], aggregates formed by elliptic particles are studied under shear flow.

Among the numerical methods, the Arbitrary Lagrange Euler (ALE) [5] method and the distributed Lagrange multiplier based fictitious domain method (FDM) [6-8] are presently the two most successful approaches for the simulation of the interaction between the moving objects and the fluid flow. The ALE-based finite element method has its advantages over the traditional Lagrange-based finite element formulation (a prior fixed system in space) and the Eulerian-based finite element formulation (a system attached to material). However, this moving grid method exhibits its disadvantages such as the distortion of the mesh and the need for re-meshing. In contrast to the ALE, using the FDM, the solution of the problem posed in such a complex-shaped and time-dependent domain is reduced to a problem of the same nature in a rectangular domain which contains the former domain. The new computational domain is fixed and time-independent. Thus fixed mesh can be used and for which high-performance solution methods are available. The FDM has been widely used for the solution of the problems arising in the biomechanical and biomedical field. For instance, this technique has been applied to heart valve modeling $[9,10]$.

In the present study, the simulation of the motion of multiple RBCs in a microvessel using the FDM is discussed. The cells are modeled as solid neutrally buoyant (NB) particles with biconcave shapes. The aggregability of the cells is described by a depletion interaction model [11]. Since biconcave particles have not been simulated using this method, and the FDM has not been applied to the simulation of RBC aggregation previously, the objectives in this study are (i) to construct an algorithm to simulate the flow of particles at a low Reynolds number (Re) using the FDM, (ii) to apply the method to study the motion of multiple non-aggregating and aggregating biconcave particles in a microchannel, and (iii) to demonstrate its feasibility for simulating the fluid-particle interaction in blood flow.

\section{Mathematical models}

\subsection{Blood flow model}

Blood is viewed as a fluid-particle system in which blood plasma is assumed to be an incompressible and Newtonian fluid and the blood cells are assumed to be NB particles. It is also assumed that the lubrication forces are large enough to prevent RBCs from touching the fixed vessel walls and there exists an interacting force between two cells. The domain occupied by the fluid and the particles are denoted as $\Omega_{f}$ and $\Omega_{p}$, respectively. Points in the domains are denoted by $\mathbf{x}=\left(x_{1}, x_{2}\right)$. For some time $T>0$, the equations of motion of the plasma are therefore

$$
\begin{array}{r}
\rho_{f}\left[\frac{\partial \mathbf{u}}{\partial t}+(\mathbf{u} \cdot \boldsymbol{\nabla}) \mathbf{u}\right]=\rho_{f} \mathbf{g}+\boldsymbol{\nabla} \cdot \boldsymbol{\sigma} \text { in } \Omega_{f}, t \in(0, T), \\
\boldsymbol{\nabla} \cdot \mathbf{u}=0 \text { in } \Omega_{f}, t \in(0, T),
\end{array}
$$

where $\mathbf{u}, \rho_{f}$, and $\mathbf{g}$ are the flow velocity, density of the fluid, and gravity, respectively. For a fluid of viscosity $\mu_{f}$ and pressure $p$, the stress tensor is expressed as $\sigma=-p \mathbf{I}+2 \mu_{f} \mathbf{D}(\mathbf{u})$ with the identity matrix 
I and the deformation rate tensor $\mathbf{D}(\mathbf{u})=\left(\boldsymbol{\nabla u}+(\boldsymbol{\nabla u})^{T}\right) / 2$. The motion for each particle is given by the Euler-Newton's equations, namely

$$
\begin{array}{r}
\mathbf{v}(\mathbf{x}, t)=\mathbf{V}(t)+\omega(t) \times \overrightarrow{\mathbf{G}(t) \mathbf{x},} \\
\frac{d \mathbf{G}}{d t}=\mathbf{V}, \\
m \frac{d \mathbf{V}}{d t}=m \mathbf{g}+\mathbf{F}^{h}+\mathbf{F}^{r}, \\
\frac{d\left(\mathbf{I}_{p} \omega\right)}{d t}=\mathbf{T}^{h}+\overrightarrow{\mathbf{G} \mathbf{x}_{r}} \times \mathbf{F}^{r},
\end{array}
$$

where $m, \mathbf{V}, \mathbf{I}_{p}$, and $\omega$ are the mass, velocity of the center of mass, moment of inertia, and angular velocity of the RBC particle, respectively. $\mathrm{x}$ is the position vector from the center of mass $\mathbf{G}$ to the surface of the cell and $\overrightarrow{\mathbf{G x}}=(\mathbf{x}-\mathbf{G})$. In Eq. (5), $\mathbf{F}^{r}=\mathbf{F}^{a}+\mathbf{F}^{w}$ where $\mathbf{F}^{a}$ is the interaction force acting on the particle when two RBCs are close to each other and $\mathbf{F}^{w}$ is the short-range repulsive force in the case of cell-boundary interaction [12]. Then a torque in Eq. (6) acts on the point $\mathbf{x}_{r}$ where $\mathbf{F}^{r}$ applies on the particle. The hydrodynamic force $\mathbf{F}^{h}$ exerted by the fluid on the particle is in the form $\mathbf{F}^{h}=-\int_{\gamma} \boldsymbol{\sigma} \mathbf{n} d \gamma$, and the torque (about the center of the mass) exerted by the fluid on the particle is $\mathbf{T}^{h}=-\int_{\gamma} \overrightarrow{\mathbf{G x}} \times \boldsymbol{\sigma} \mathbf{n} d \gamma$, with $\mathbf{n}$ the unit normal vector pointing out of the particle. The boundary conditions are such that at the inlet and outlet of the channel, periodic boundary conditions are applied; at the vessel wall, $\Gamma_{w}$, the no-slip condition is assumed, i.e., $\left.\mathbf{u}\right|_{\Gamma_{w}}=\mathbf{0}$, and the no-slip boundary conditions for rigid boundaries are that the fluid at $\partial \Omega_{p}$ must move with the prescribed boundary motions, namely $\left.\mathbf{u}\right|_{\partial \Omega_{p}}=\left.(\mathbf{v}+\omega \times \overrightarrow{\mathbf{G x}})\right|_{\partial \Omega_{p}}$.

\subsection{RBC aggregation model}

In two-dimensional (2D) simulations, the biconcave shape of the RBC is approximated by the characteristic cross section in the plane that is parallel to the flow direction if the cell were in shear flow. In this study, the cross section of the particle is assumed to have a uniform biconcave shape as described in [13] by the scaled equation:

$$
\bar{y}=0.5\left(1-\bar{x}^{2}\right)^{1 / 2}\left(a_{0}+a_{1} \bar{x}^{2}+a_{2} \bar{x}^{4}\right)
$$

where $\bar{x}=x / d, \bar{y}=y / d, d$ is the diameter of the cell and $a_{0}=0.207, a_{1}=2.002, a_{2}=-1.122$. The interaction forces between two particles have been regarded as fundamental to the understanding of aggregation of the RBCs. Until recently, the mechanism underlying the RBC aggregation process remains unclear. In the present study, A Morse type potential function proposed in [11] for RBC adhesion phenomena has been used to describe the intercellular energy between two cells due to its simplicity:

$$
\phi(r)=D_{e}\left[e^{2 \beta\left(r_{0}-r\right)}-2 e^{\beta\left(r_{0}-r\right)}\right]
$$




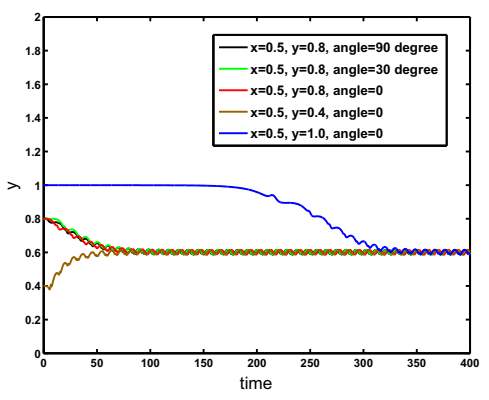

Fig. 1. Trajectory of the cell center of biconcave particles in a pressure-driven Poiseuille flow which shows the lateral migration of the particles.

where $r$ is the distance between two points considered, $r_{0}$ and $D_{e}$ are reference distance and surface energy, respectively, and $\beta$ is a scaling factor. The intercellular force is then $f(r)=-\partial \phi / \partial r$. Employing this potential function, the intercellular force is attractive at far distances and repulsive at near distances. The behavior of the function qualitatively represents the characteristics of the interaction between the RBCs, which shows that it is consistent with the depletion energy model. Because multiple cells were included in the study, a cut-off circle was specified to identify the effective region of the intercellular force. The interaction force acting on a cell is then the sum of the forces generated by the neighboring cells in the effective domain.

\section{Numerical simulation}

\subsection{Lateral migration}

Prior to the numerical studies, the method was validated by considering the problem of lateral migration of the biconcave particles in a pressure-driven Poiseuille flow. At five different initial vertical position and/or tilted angles (about $y$-axis) of the long axis of the RBC, simulations were carried out in a Newtonian fluid. In the validation, the parameters were in scaled form. The diameter of the particle was chosen as $d=0.5$. For simplicity, the fluid density was chosen to be $\rho=1.0$ and the fluid viscosity $\mu=0.1$. The computational domain was $\Omega=(0,12) \times(0,2)$. The mesh size for the velocity field was $h=1 / 64$. Figure 1 shows that all the rigid particles of biconcave shape moved to the same equilibrium position, which is about 0.6 in the $y$-direction and away from the central axis. At time $t=500$, the average particle speed was 1.6177 ; hence the particle $R e$ was 8.139 . The results qualitatively agree well with the lateral migration of the NB circular particles reported in [7].

\subsection{Simulation of four cells}

Two cases for the motion of four RBCs in Poiseuille flow were studied, reflecting different initial configurations of the RBCs. The results of four cells without aggregating force to that of the same cells with aggregating force are compared in each case.

Figure 2 shows the snapshots of four non-aggregating cells moving downstream in the Poiseuille flow. The cells were initially positioned vertically in the fluid with center-to-center distance $3 \mu \mathrm{m}$. Since the center of mass of the particles were initially located at the centerline, where shear force is almost negligi- 

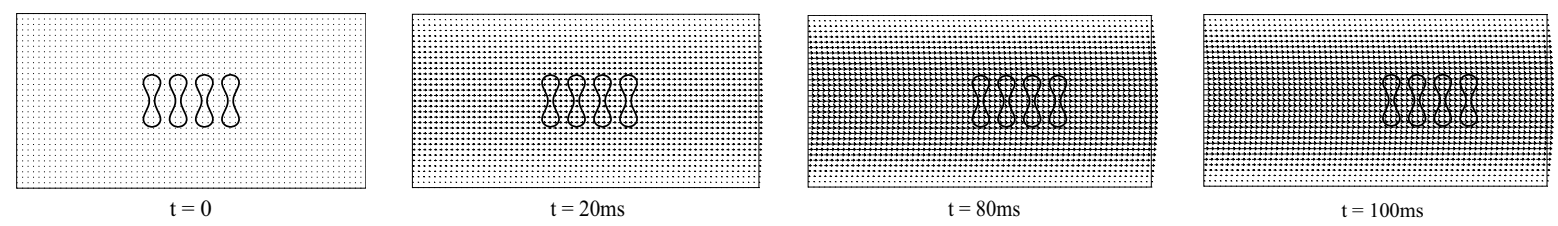

Fig. 2. Snapshots of motion of four biconcave NB particles without attractive force, maximum fluid velocity at $t=100 \mathrm{~ms}$ is $v=0.09 \mathrm{~cm} / \mathrm{s}, R e=0.01$.
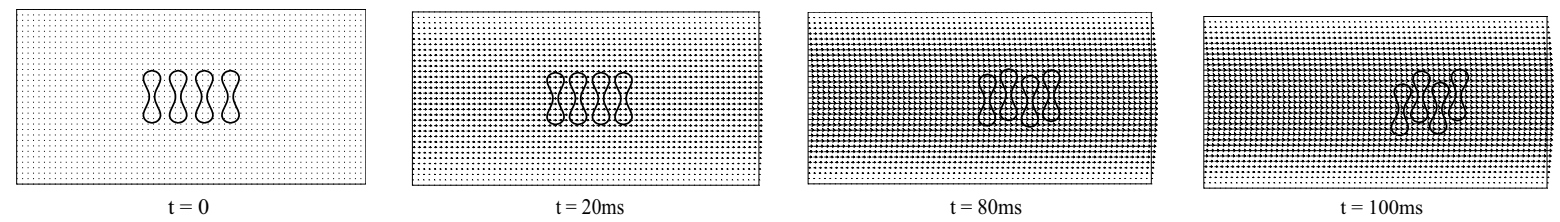

Fig. 3. Snapshots of motion of four biconcave NB particles with attractive force, $D e=1 \times 10^{-5} \mu \mathrm{J} / \mu \mathrm{m}^{2}, \beta=3 / \mu \mathrm{m}$, $r_{0}=1 \mu \mathrm{m}$, maximum fluid velocity at $t=100 \mathrm{~ms}$ is $v=0.09 \mathrm{~cm} / \mathrm{s}, R e=0.01$.
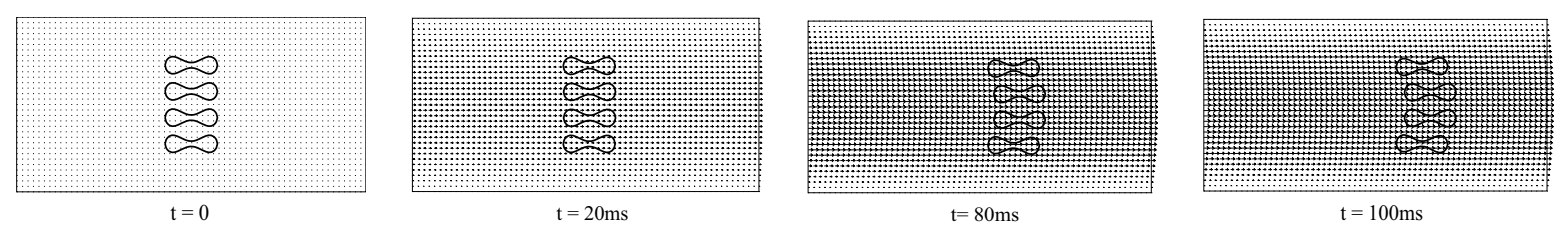

Fig. 4. Snapshots of motion of four biconcave NB particles without attractive force, maximum fluid velocity at $t=100 \mathrm{~ms}$ is $v=0.09 \mathrm{~cm} / \mathrm{s}, R e=0.01$.

ble, the cells moved downstream without noticeable rotation and the distance between particles remained almost unchanged. However, unlike the case for non-aggregating RBCs, the attractive force between the cells increased the speed of the upstream cells and decreased the downstream cells. As a result, the upstream cells caught up with the downstream cells. When the cells were close enough, the intercellular force came into effect. Figure 3 shows that due to the attractive force and repulsive force cells moved slightly off the centerline of the channel. However, in this case, the cells did not attaining an equilibrium configuration. The position of each cell in the rouleaux changed with time as they were moving together in the fluid.

As a second case, the motion of four cells, which were initially placed horizontally with center-tocenter $3 \mu \mathrm{m}$ was computed. When the cells were non-aggregating, the RBCs moved separately in the flow (Figure 4). The cells away from the centerline of the domain moved slower than the cells close to the centerline because the velocity profile of the fluid is nearly parabolic. When the cells were aggregating, the cells approached each other because of the attractive force as shown in Figure 5. Due to the balance of the hydrodynamic force and the cell-cell interaction, the two middle cells moved a little faster than the two outside cells, finally attaining an equilibrium position and moving together as a single compact aggregate.

\subsection{Simulation of thirty cells}

To achieve a RBC concentration close to the hematocrit of human blood (around 40-45\%), 30 RBCs were put in a fluid domain of size $(0,40) \times(0,16) \mu \mathrm{m}^{2}$, corresponding to a hematocrit around $37.6 \%$. 


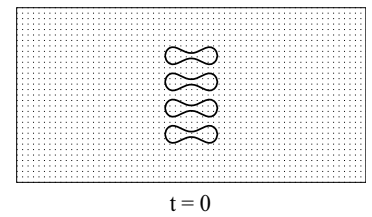

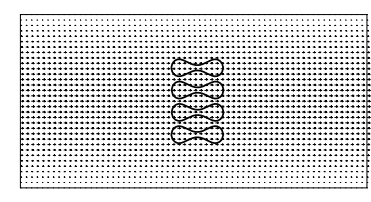

$\mathrm{t}=20 \mathrm{~ms}$

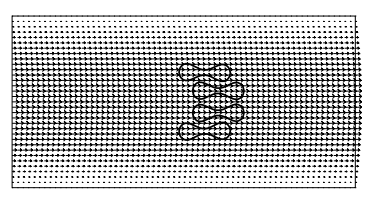

$\mathrm{t}=80 \mathrm{~ms}$

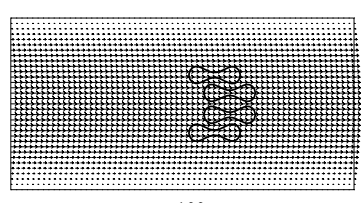

$\mathrm{t}=100 \mathrm{~ms}$

Fig. 5. Snapshots of motion of four biconcave NB particles with attractive force, $D e=1 \times 10^{-5} \mu \mathrm{J} / \mu \mathrm{m}^{2}, \beta=3 / \mu \mathrm{m}$, $r_{0}=1 \mu \mathrm{m}$, maximum fluid velocity at $t=100 \mathrm{~ms}$ is $v=0.09 \mathrm{~cm} / \mathrm{s}, R e=0.01$.
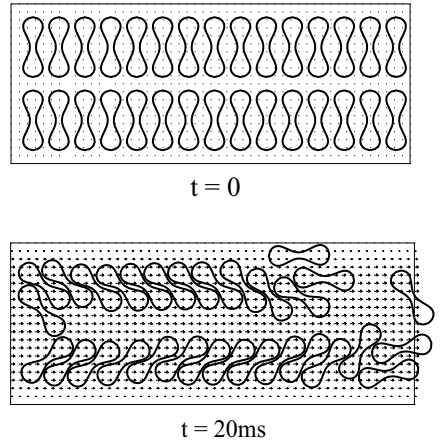
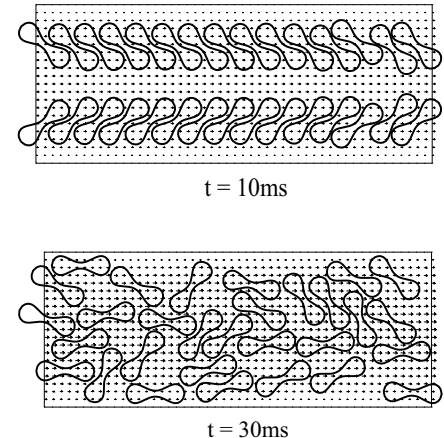

Fig. 6. Snapshots of motion of 30 biconcave NB particles without aggregating force, maximum fluid velocity at $t=30 \mathrm{~ms}$ is $v=3 \mathrm{~cm} / \mathrm{s}, R e=0.201$.
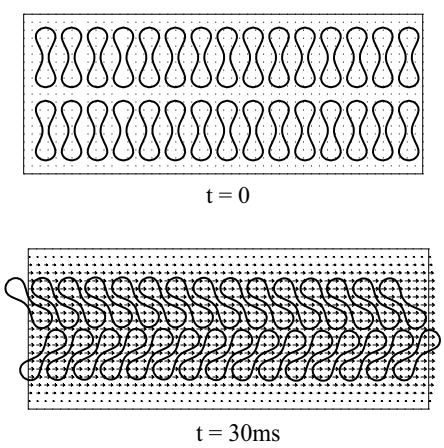
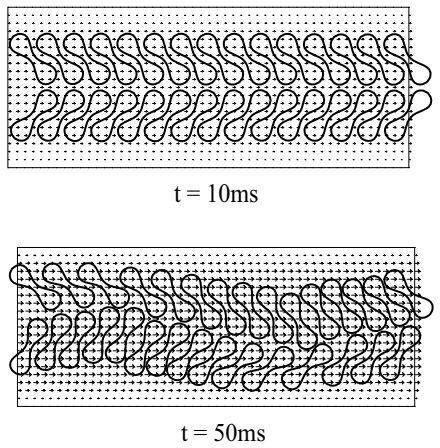

Fig. 7. Snapshots of motion of 30 biconcave NB particles with aggregating force, $D e=1 \times 10^{-5} \mu \mathrm{J} / \mu \mathrm{m}^{2}, \beta=3 / \mu \mathrm{m}$, $r_{0}=1 \mu \mathrm{m}$, diameter $d=6 \mu \mathrm{m}$, maximum fluid velocity at $t=50 \mathrm{~ms}$ is $v=3 \mathrm{~cm} / \mathrm{s}, R e=0.267$.

The initial configuration of these $30 \mathrm{RBCs}$ was such that two files of the cells were placed vertically in the channel with equal spacing. The snapshots of simulations for non-aggregating RBCs and aggregating RBCs are shown in Figures 6 and 7, respectively. As can be seen in these figures, the non-aggregating cells were dispersed by the hydrodynamic forces exerted by the flow. As time elapsed, the concentration of the cell at the centerline became higher than the cell concentration close to the wall. On the other hand, for the aggregating cells, the attractive force would keep them together and form rouleaux as they were moving in the flow. For the parameters used in this study, this force was strong enough to bind the cells together for sufficient long simulation time. The vector fields of the flow are also presented in Figures 6 and 7. 


\section{Conclusion}

It has been recognized that numerical investigations of RBC aggregation at the cellular level is of physiological and clinical significance. In this study, the application of distributed Lagrange multiplier based fictitious domain method for fluid-particle interaction for a particulate flow was presented. The motion and aggregation of multiple RBCs in a microchannel at periodic boundary conditions at the inlet and the outlet was investigated. The method has been validated for lateral migration of the biconcave particles in plane Poiseuille flow. A comparison of the results for multiple cells without and with aggregation is presented. It is clear that when there is aggregating force, the motion of the cells depends on more than the flow field around them. Apparently, the motion and configuration of the RBC aggregates is a balance of the hydrodynamic force and intercellular force and is associated with the initial position of the cells. From the visualization of the vector fields and the motion and aggregation of RBCs, it can be concluded that DLM in an effective and important numerical tool for the simulation of the fluid-particle interactions without assuming the motion of the particles in advance. It is shown that the multiple complex geometries are easily treated with fixed uniform mesh using the fictitious domain method. Moreover, the algorithm has the modular nature so that it can be applied to a large class of problems involving the processes of cell aggregation and fluid-cell interaction. In future study, the flow domain can be more complex than merely a straight channel, including curved and bifurcating vessels. In addition, the simulation presented here is two-dimensional, and the methodology has the potential to be further extended to three-dimensional studies.

\section{References}

[1] T. Wang, T.-W. Pan, Z.W. Xing and R. Glowinski, Numerical simulation of rheology of red blood cell rouleaux in microchannels, Physical Review E 79 (2009), 041916.

[2] M. Fenech, D. Garcia, H.J. Meiselman and G. Cloutier, A particle dynamic model of red blood cell aggregation kinetics, Annals of Biomedical Engineering 37 (2009), 2299-2309.

[3] J.K.W. Chesnutt and J.S. Marshall, Structural analysis of red blood cell aggregates under shear flow, Annals of Biomedical Engineering 38 (2010), 714-728.

[4] D. Xu, E. Kaliviotis, A. Munjiza, E. Avital, C. Ji, and J. Williams, Large scale simulation of red blood cell aggregation in shear flows, Journal of Biomechanics 46 (2013), 1810-1817.

[5] J. Donea, S. Giuliani and J.P. Halleux, An arbitrary Lagrange-Eulerian finite element method for transient dynamic fluidstructure interactions, Computer Methods in Applied Mechanics and Engineering 33 (1982), 689-723.

[6] L.H. Juarez, R. Glowinski and B.M. Pettitt, Numerical simulation of sedimentation of a Tripole-like body in an incompressible viscous fluid, Applied Mathematics Letters 15 (2002), 743-747.

[7] T.-W. Pan and R. Glowinski, Direct simulation of the motion of neutrally buoyant circular cylinders in plane Poiseuille flow, Journal of Computational Physics 181 (2002), 260-279.

[8] T.-W. Pan, C.-C. Chang and R. Glowinski, On the motion of a neutrally buoyant ellipsoid in a three-dimensional Poiseuille flow, Computer Methods in Applied Mechanics and Engineering 197 (2008), 2198-2209.

[9] J. De Hart, G.W.M. Peters, P.J.G. Schreurs and F.P.T. Baaijens, A three-dimensional computational analysis of fluidstructure interaction in the aortic valves, Journal of Biomechanics 36 (2003), 103-112.

[10] R. Van Loon, P.D. Anderson, J. De Hart and F.P.T. Baaijens, A combined fictitious domain/adaptive meshing method for fluid-structure interaction in heart valves, International Journal for Numerical Methods in Fluids 46 (2006), 533-544.

[11] Y. Liu, L. Zhang, X. Wang and W.K. Liu, Coupling of Navier-Stokes equations with protein molecular dynamics and its application to hemodynamics, International Journal for Numerical Methods in Fluids 46 (2004), 1237-1252.

[12] M. R. king and D. A. Hammer, Multiparticle adhesive dynamics. Interactions between stably rolling cells, Biophysical Journal 81 (2001), 799-813.

[13] Y. Liu and W.K. Liu, Rheology of red blood cell aggregation by computer simulation, Journal of Computational Physics 220 (2006), 139-154. 